Ю.Л.Виншток ${ }^{1}$, К.Э.Гулиикий ${ }^{1}$, Г.Б.Зеленин ${ }^{2}$

\title{
Новая методика неинвазивного обследования легких на основе применения Vibration Response Imaging
}

1 - компания Deep Breeze: 2 Hailan St. P0 Box 140, North Industrial Park, Or-Akiva, 30600 Israel;

2 - компания "Росслин Медикал": 123610, Москва, Краснопресненская наб., 12, оф. 1708

\section{Yu.L.Vinshtok, K.E.Gulitsky, G.B.Zelenin \\ Novel technique of non-invasive lung investigation with Vibration Response Imaging}

Key words: Vibration Response Imaging (VRI), vibration energy, lung sounds, Quantitative Lung Data (QLD), regional distribution of lung sounds, envelope of acoustic signals (EVP), Deep Breeze.

Ключевые слова: вибрационная диагностика и визуализация легких, энергия вибрации, легочные звуки, количественная оценка легочных звуков, региональная оценка легочных звуков, энергетическая огибающая акустического сигнала, Deep Breeze.

Аускультация легочных звуков является важным элементом объективного осмотра больного. Со времени изобретения Р.Лаэннеком стетоскопа в 1816 г. аускультация обеспечивает врача важной клинической информацией. К сожалению, стетоскоп, прошедший многолетнюю эволюцию, несмотря на широту применения в повседневной врачебной практике, является недостаточно совершенным инструментом. Его возможности ограничены различными техническими характеристиками и степенью чувствительности, зависящими от производителя, с одной стороны, и уровнем интерпретации, обусловленной аускультативными навыками врача, - с другой $[1,2]$. Таким образом, субъективный метод обследования посредством стетоскопа может быть источником расхождений и противоречий в оценке характера легочных звуков [3]. В ряде работ [1, 4] правомерно ставится под вопрос точность и, как следствие, диагностическая ценность стетоскопа.

Появление и развитие вычислительной техники дало толчок к исследованию дыхательных звуков посредством цифровых технологий с целью разработки методики обследования легких, которая могла бы давать объективные, точные, воспроизводимые данные, характеризующие с высокой вероятностью физиологическое состояние дыхательной системы.

Новые поколения компьютеров, позволяющие значительно ускорить процесс обработки информации, дали возможность перейти от "компьютеризированного стетоскопа" к оценке физиологического состояния легких на основе мультифокального анализа. Пространственную информацию, полученную с помощью легочных звуков, записанных одновременно в различных местах грудной клетки, можно оценить с помощью методов визуализации акустической информации [5]. Анализ этих данных позволяет расширить знания о патофизиологии протекающего респираторного заболевания, которые могут применяться в клинической практике [6].

\section{Технология Vibration Response Imaging}

Vibration Response Imaging (VRI - технология вибрационной диагностики и динамической визуализации легких) является новейшим методом визуальной и количественной оценки акустических сигналов легких. Она основана на регистрации вибрации, возникающей при прохождении воздуха по проводящим путям легких, и последующей компьютерной обработке полученных данных с целью построения динамических изображений.

В основе разработки вибрационной диагностики и динамической визуализации легких лежит общепринятая концепция возникновения легочных звуков. Инспираторные легочные звуки появляются вследствие турбуленции воздушных потоков на уровне лобарных или сегментарных бронхов [1, 6], в то время как экспираторные звуки - на более проксимальном уровне вследствие интраламинарных потоков [1]. Генерируемая при этом энергия вибрации после прохождения и фильтрации через паренхиму легких и окружающие ткани передается на поверхность грудной клетки [6], где регистрируется с помощью датчиков. Параметры записываемой с поверхности тела вибрации зависят от структурных и функциональных особенностей легких, а также имеют различный характер при вдохе и выдохе [1].

\section{Система акустической визуализации}

Система VRIxp (Deep Breeze Ltd., Израиль) является коммерческой системой на основе VRI (рис. 1). Она предназначена для компьютеризированного обследования легких без радиационного облучения.

Аппаратное обеспечение системы VRI-XP представляет собой 2 матрицы ( -arrays), каждая из которых состоит из 20 пьезоэлектрических контактных сенсоров (Meditron $A S A$, Норвегия) с линейным частотным откликом в диапазоне частот 50-400 Гц 


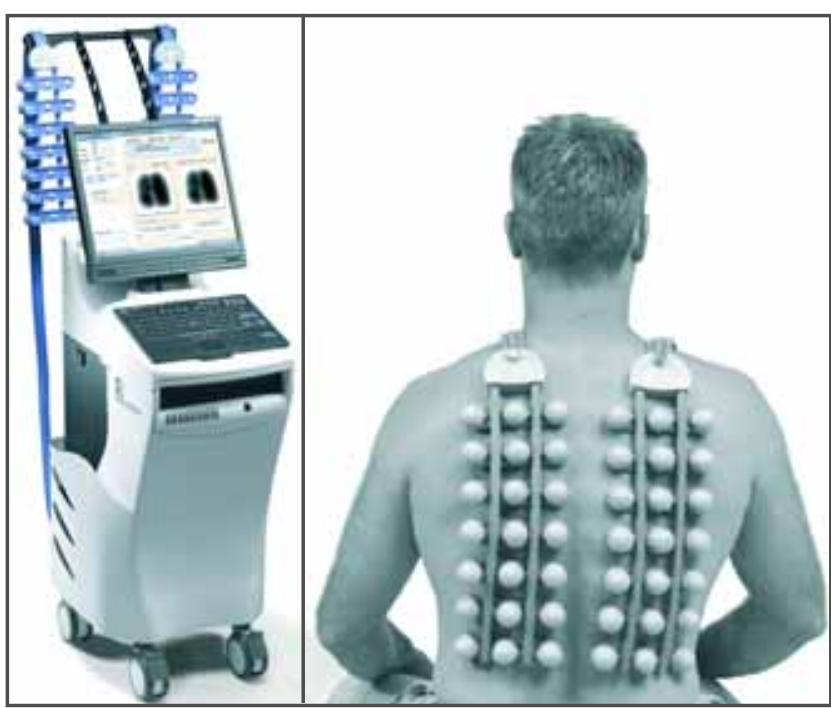

Рис. 1. Система VRIxp

Рис. 2. Матрицы, закрепленные на спине пациента посредством вакуума

с точностью \pm 2 дБ. Расстояние между центрами сенсоров -4 см по горизонтали и 5 см по вертикали. Сенсоры закрепляются на спине пациента с помощью слабого вакуума, постоянная величина которого во время записи контролируется системой (рис. 2).

Записанные как цифровой сигнал данные дыхания представляются в виде кривой изменения его интенсивности во времени. Для уменьшения помех, связанных с движением грудной клетки и межреберных мышц, а также сердечных тонов, применяются фильтры в диапазоне 150-250 Гц. Обработанные сигналы объединяются и делятся на отдельные кадры с интервалом 0,17 с для создания динамического изображения (рис. 3). Анализ сигналов включает в себя использование 4 алгоритмов: 1) вычисление изменения интенсивности сигналов во времени и вычисление динамической шкалы интенсивности энергии посредством градации серого цвета; 2) обнаружение влажных хрипов; 3) обнаружение сухих хрипов; 4) региональная оценка функционирования легких. Это позволяет системе VRIxр не только произвести динамическую визуализацию всего дыха-

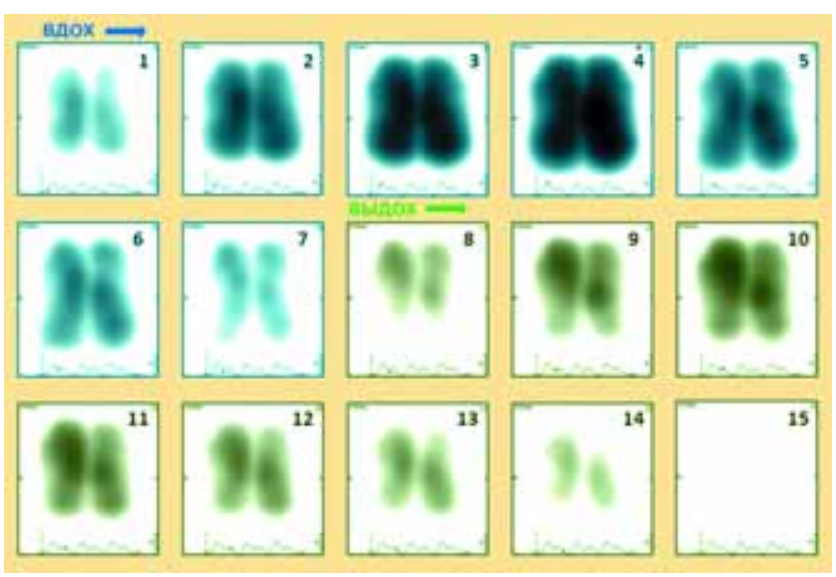

Рис. 3. Покадровое изображение дыхательного цикла (вдох-выдох)

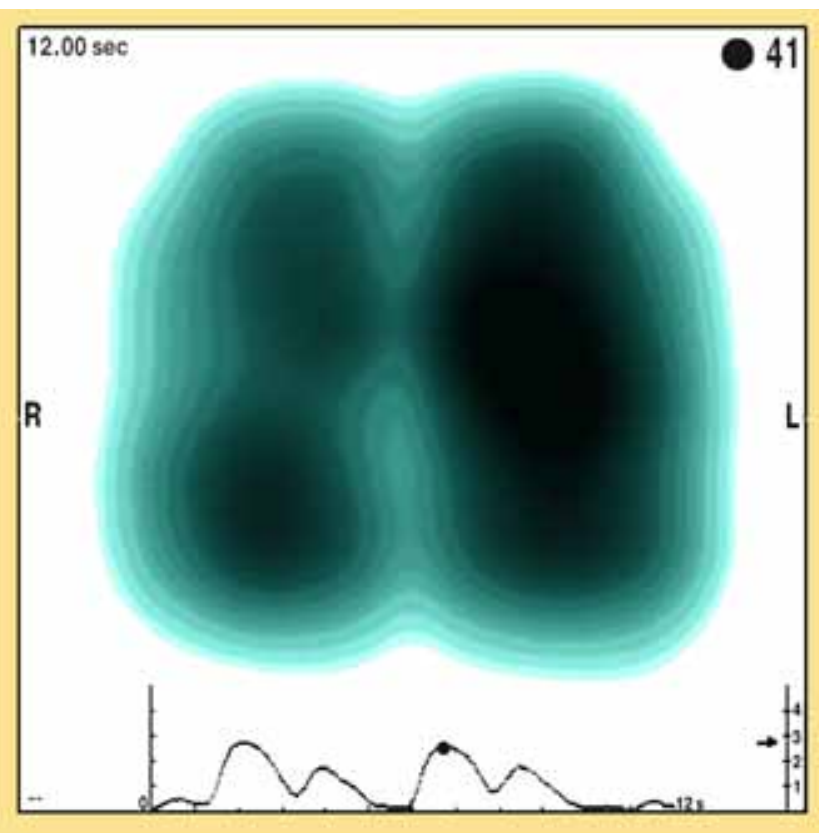

Рис. 4. Кадр максимальной энергии

тельного цикла и автоматически распознать и локализовать хрипы, но и количественно оценить полученные данные и, как результат, дать оценку состояния легких в реальном времени.

Результаты вибрационной диагностики и динамической визуализации легких могут быть представлены в нескольких вариантах: динамическое изображение (в режиме кинопетли или в режиме "кадр за кадром"), отдельное изображение в момент максимальной энергии вибраций (кадр МЕF - maximum energy frame), график энергии вибрации и таблица количественных данных (региональное распостранение энергии вибрации). Результаты проведенной записи пациента могут быть сохранены на жестком диске системы или загружены на компакт-диск или флэш-накопитель, а затем распечатаны для истории болезни.

\section{Воспроизведение динамического изображения}

Графическое изображение, выраженное в виде серой шкалы интенсивности сигналов, отфильтрованных в выбранном диапазоне частот и изменяющихся по времени, является динамической картиной VRI. Энергия вибрации, полученная во время записи, "нормализуется" и результат отражается на шкале интенсивности от 0 до 4. Энергия вибрации высокой интенсивности представлена темными цветами от темно-серого до черного, энергия вибрации низкой интенсивности - светло-серым, а отсутствие вибрации - белым цветом.

Системный интерфейс позволяет производить выбор и оценку статического изображения в любой точке вдоха и выдоха. Изображение, полученное на пике вдоха (рис. 4), представляет собой распределение энергии вибрации дыхательного цикла при максимальном значении ее средней величины и называется кадром MEF. 


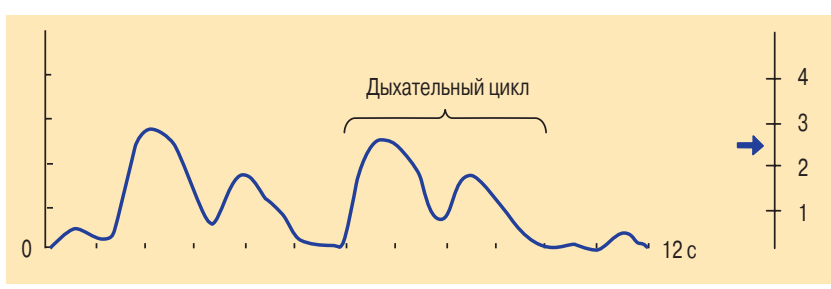

Рис. 5. График вибрационной энергии

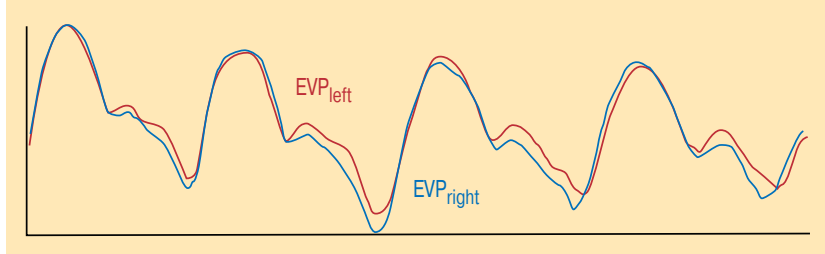

Рис. 6. График ЕVP

\section{График энергии вибрации}

Динамическая картина дополнительно включает в себя график средней энергии вибрации всех сенсоров. Он представляет собой графическое изображение изменения интенсивности энергии вибрации по линейной шкале времени за период записи. Каждый дыхательный цикл представлен, как правило, 2 пиками - вдоха и выдоха (рис. 5). При анализе графика значение имеют значение продолжительность дыхательного цикла в целом, взаимоотношение фазы вдоха и выдоха, общий уровень интенсивности сигнала, уровень интенсивности на вдохе и выдохе и форма графика. Кроме того, система позволяет анализировать кривую, являющуюся энергетической огибающей записанного сигнала (EVP). Для анализа используются средние величины EVP сигналов (рис. 6), полученных от левой (EVP $\left.\mathrm{P}_{\text {left }}\right)$ и правой $\left(\mathrm{EVP}_{\text {right }}\right)$ матриц.

\section{Количественная региональная оценка}

Область каждого легкого делится на 3 региона в соответствии с группами микрофонов: 2 верхних ряда микрофонов составляют верхний, 2 средних - средний и 2 нижних - нижний регион. Система позволяет определить относительную величину интенсивности вибрации каждого региона легких к суммарной интенсивности всех сигналов (100\%) в процентном соотношении. После получения данных алгоритм выделяет относительный уровень энергии каждого региона из общего уровня вибрационной энергии. Информация по региональному распределению ин-

Таблица Данные QLD

\begin{tabular}{|l|c|c|}
\hline & Right, $\%$ & Left, \% \\
\hline Upper & 12 & 17 \\
\hline Middle & 15 & 19 \\
\hline Lower & 18 & 19 \\
\hline TOTAL & 45 & 55 \\
\hline
\end{tabular}

тенсивности энергии вибрации представлена в виде таблицы процентных соотношений, схожей с таблицей количественных результатов вентиляционноперфузионной сцинтиграфии. Эти данные определяются как quantitative lung data, или QLD (таблица).

\section{Обнаружение влажных и сухих хрипов}

Влажные хрипы акустически представляют собой короткие прерывистые звуки, характеризующиеся графически внезапным резким подъемом интенсивности записанного сигнала с последующим более плавным снижением [1]. VRI не делает различия между разными типами влажных хрипов и выявляет их на графике сигнала как максимальные пики, соответствующие морфологическим параметрам, описанным N.Gavriely [7]

Сухие хрипы характеризуются акустически синусоидальной формой сигнала и плавным изменением несущей частоты в направлении высоких частот [1]. Алгоритм распознавания сухих хрипов в VRI обрабатывает записанный сигнал посредством метода дигитального разложения Фурье. Максимальные пики в диапазоне частот 100-700 Гц оцениваются в соответствии с эмпирическими спектральными критериями, сформулированными N.Gavriely [7].

\section{Повторяемость результатов}

Повторяемость результатов является предварительным необходимым условием разработки клинического метода визуализации дыхательных звуков. N.Gavriely и M.Mahaghan [8] показали, что при повторных записях спектральные характеристики звуков дыхания на вдохе и выдохе существенно не различаются. В свою очередь, T.M.Maher et al. [9] посредством технологии VRI обнаружили, что у здоровых индивидуумов с течением времени количественные показатели дыхания (включая интенсивность сигналов, отраженных графически) также значительно не изменяются. Анализ был проведен при 1-сторонней и перекрестной оценке записей, полученных через различные промежутки времени. M.Yigla et al. [10] выявили отличительные и предсказуемые особенности, присущие визуальной динамической картине дыхания здоровых индивидуумов и подтвержденные количественными данными. Эти характерные особенности составляют стандартную динамическую картину VRI, используемую для сравнения с динамической картиной при различных легочных патологиях.

\section{Обзор литературы}

Вслед за работами, подтверждающими повторяемость VRI картины у здоровых пациентов, были проведены исследования, целью которых было выявить возможности системы при различных легочных заболеваниях. R.Mor et al. [11] использовали визуализацию распространения дыхательных звуков как конкретный и чувствительный метод дифференциации 
между здоровыми легкими и легкими при пневмонии и плевральном выпоте. Клинические исследования D.Anantham et al. [12] демонстрируют высокую степень соответствия данных VRI и рентгенографии в определении плеврального выпота.

При анализе динамической картины и графика изменения энергии вибрации дыхательных звуков пациентов с острым приступом бронхиальной астмы (БА), по сравнению со здоровыми, R.P.Dellinger et al. [13] выявили, что сужение дыхательных путей сопровождается снижением интенсивности дыхательных звуков на вдохе и повышением - на выдохе. Использование VRI при остром приступе БА демонстрирует также значительную асинхронность в распространении дыхательных звуков между правым и левым легкими, подтвержденную как динамической картиной, так и при сравнении кривых EVP. После применения бронхолитиков асинхронность значительно снижается на фоне улучшения клинического состояния [14]. В работе K.K.Guntupalli et al. [15] выявлены возможности применения VRI для дифференциальной диагностики между БА и хронической обструктивной болезнью легких (ХОБЛ) с точностью $85 \%$, по сравнению с клинической диагностикой. Качественные и количественные (региональная оценка) данные, полученные с помощью этой методики, позволяют определить более четкие различия между этими заболеваниями и применять VRI для оптимизации и прогнозирования результатов лечения.

Снижение интенсивности легочных звуков является устойчивым индикатором обструктивной болезни и обструкции воздушных путей инородным телом при 1-стороннем проявлении или в сочетании с наличием сухих хрипов и кашля [16]. H.D.Becker et al. [17] изучали возможность использования технологии VRI при обструкции центральных дыхательных путей и для оценки результатов оперативной бронхоскопии. Было показано, что локализация источника обструкции центральных дыхательных путей оказывает влияние на расположение области снижения энергии вибрации. Исследования, проведенные L.Bentur et al. [18], продемонстрировали возможности системы VRI при подозрении на аспирацию инородного тела в педиатрической практике. В работе M.Blanco et al. [19] демонстрируется использование динамической картины VRI для пневмоторакса, при котором она характеризуется снижением интенсивности энергии вибрации в пораженной области легкого в течении всего дыхательного цикла.

S.Jean et al. [20] описали влияние ошибочной 1-сторонней интубации легких на распределение интенсивности дыхательных звуков. Результаты работы демонстрируют значительную диспропорцию между вентилируемым и невентилируемым легким, что позволяет рассматривать VRI как дополнительный быстрый и эффективный метод подтверждения правильного размещения эндотрахеальной трубки. I.Cinel et al. [21] описали возможности применения данной методики при ошибочной интубации пищевода.

Определение показаний к резекции легких, особенно при наличии опухоли, зависит от прогнозиро- вания их послеоперационной функциональности, т. к. хроническая обструкция воздушного потока часто сопутствует раку легких. В таких случаях рекомендуется перфузионная сцинтиграфия. Недавнее исследование R.C.Morice et al. [22] показало отсутствие значительной разницы между методикой VRI и сцинтиграфией для прогноза функциональности легких после хирургического лечения. Высокая степень корреляции между VRI и сцинтиграфией также была продемонстрирована при проведении сравнительной оценки состояния пересаженного легкого до и после 1-сторонней трансплантации легких [23].

\section{Аспекты практического применения}

Суммируя результаты вышеупомянутых исследований, следует остановиться на аспектах применения VRI в практической медицине.

1. Аспект массовости. Методика дает возможность производить скрининг больших групп населения благодаря быстрой и простой процедуре записи пациента.

2. Экономический аспект. Отсутствие необходимости в специальном помещении и расходных материалах, связанных с эксплуатацией прибора, а также его мобильность позволяют использовать аппаратуру почти повсеместно со значительным экономическим эффектом по сравнению с другими визуальными методами обследования.

3. Аспект безопасности. Методика не подразумевает применения рентгеновского или какого-либо другого излучения, а использует вибрацию, возникающую естественным путем. Правомочно говорить о полной безопасности данной методики как для пациентов, так и для медицинского персонала. Процедура записи может быть повторена многократно в течение различных промежутков времени. Думается, вибрационная диагностика с динамической визуализацией легких является единственным визуальным методом обследования легких беременных женщин, безопасным для плода.

4. Клинический аспект. На основании проведенных исследований и накопленного опыта в настоящее время методика может применяться в практической медицине в нескольких направлениях:

- дополнительная методика диагностики легочных заболеваний;

- легкий и дешевый способ мониторинга эффективности терапии при острых и хронических состояниях (БА, ХОБЛ, пневмония, плевральный выпот, пневмоторакс);

- контроль эффективности и диагностика осложнений при бронхоскопии и интервенционных бронхоскопических процедурах;

- оценка функциональности при операциях на легких, включая трансплантацию;

- диагностика инородных тел дыхательных путей, не визуализируемых другими методами;

- оценка состояния легких курящих пациентов. 


\section{Заключение}

Являясь новейшей методикой визуализации и количественной оценки легочных звуков, VRI объединяет в себе новейшие достижения компьютерных технологий и известные принципы обследования легких. Отсутствие облучения и неинвазивность технологии в сочетании с удобством и простотой применения системы VRIxр дают дополнительный качественный инструмент для выявления, диагностики, мониторинга и изучения легочных заболеваний.

Авторы благодарят компанию "Росслин Медикал" за помощь в подготовке статьи. Компания "Росслин Медикал" (www. rosslynmedical.com) является экслюзивным дистрибьютором системы VRIxр в России и странах СНГ.

\section{Литература}

1. Postercamp H., Kraman S.S., Wodicka G.R. Respiratory sounds. Advances beyond the stethoscope. Am. J. Respir. Crit. Care Med. 1997; 156 (3): 974-987.

2. Abella M., Formolo J., Penney D.G. Comparison of the acoustic properties of six popular stethoscopes. J. Acoust. Soc. Am. 1992; 91: 2224-2228.

3. Loudon R.G. The lung exam. Clin. Chest Med. 1987; 8 (2): 265-272.

4. Wipf J.E., Lipsky B.A., Hirschman J.V. et al. Diagnosing pneumonia by physical examination: relevant or relic? Arch. Intern. Med. 1999; 159 (10): 1082-1087.

5. Kompis M., Pastercamp H., Wodicka G.R. Acoustic imaging of the human chest. Chest 2001; 120: 1309-1321.

6. Sovijarvi A.R.A., Malmberg L.P., Charbonneau G. et al. Characteristics of breath sounds and adventitious respiratory sounds. Eur. Respir. Rev. 2000; 10: 591-596.

7. Gavriely N., Cagell D.W. Breath sounds methodology. Los Angeles: CRC Press; 1995. 18-21.

8. Mahaghan M., Gavriely N. Repeatability of measurements of normal lung sounds. Am. J. Respir. Crit. Care Med. 1994; 149: 477-481.

9. Maher T.M., Gat M., Allen D. et al. Reproducibility of dynamically represented acoustic lung images from healthy individuals. Thorax 2008; 63: 542-548.

10. Yigla M., Gat M., Meyer J.-J. et al. Vibration Response Imaging technology in healthy subjects. Am. J. Roentgenol. 2008; 191: 845-852.

11. Mor R., Kushnir I., Meyer J.J. et al. Breathe sound distribution images of patients with pneumonia and pleural effusion. Respir. Care 2007; 52: 1753-1760.
12. Anantham D., Herth F., Majid A. et al. Vibration Response Imaging in the diagnostic evaluation of pleural effusions: A feasibility study. Respiration 2008, DOI: 10.1159/000168784.

13. Dellinger R.P., Parillo J.E., Kushnir A. et al. Dynamic visualization of lung sounds with a vibration response device: A case series. Respiration 2008; 75 (1): 60-72.

14. Wang Z., Bartter T., Baumman B.M. et al. Asynchrony between left and right lungs in acute asthma. J. Asthma 2008; 45 (7): 575-578.

15. Guntupalli K.K., Alapat P.M., Bandi V.D. et al. Evaluation of obstructive lung disease with Vibration Response Imaging. J. Asthma 2008; 45 (10): 923-930.

16. Tomanske M., Gerber A.C., Stocker S. et al. Tracheobronchial foreign body aspiration in children - diagnostic value of symptoms and signs. Swiss Med. Wkly 2006; 136: 533-538.

17. Becker H.D., Slawik M., Miyazawa T., Gat M. Vibration Response Imaging (VRI) as a new tool for outcome assessment of interventional bronchoscopy: A prospective pilot study. Respiration 2008, DOI: 10.1159/000182972.

18. Bentur L., Livnat G., Hussein D. et al. Dynamic visualization of breath sound distribution in suspected foreign body aspiration: A pediatric case series. J. Bronchol. 2007; 14: 156-161.

19. Blanco M., Mor R., Fraticelli A. et al. Breath sound distribution images of patients with pneumothorax and of healthy subjects: Diagnostic yield of Vibration Response Imaging technology. Respiration 2008, DOI: 10.1159/000181166.

20. Jean S., Cinel I., Gratz I. et al. Image-based monitoring of one-lung ventilation. Eur. J. Anaesthesiol. 2008; 20: 1-7.

21. Cinel I., Jean S., Tay C. et al. Case report: Vibration Response Imaging findings following inadvertent esophageal intubation. Can. J. Anaesth. 2008; 3: 172-176.

22. Morice R.C., Jimenez C.A., Eapen G.A. et al. Vibration Response Imaging for the prediction of post operative lung function. Chest 2007; 132 (4): 486.

23. Kramer M.R., Raviv Y., Hardoff R. et al. Regional breath sound distribution analysis in single lung transplant recipients. J. Heart Lung Transplant. 2007; 26: 1149-1154.

\section{Информация об авторах}

Виншток Юрий Львович - клинический консультант компании Deеp Breeze; тел.: +972-54-780-0279; e-mail: yuri.vinshtok@deepbreeze.com Гулицкий Константин Эдуардович - д. т. н., разработчик алгоритмов компании Deep Breeze; тел: +972-52-361-0486; e-mail: konstantin. golitski@deepbreeze.com

Зеленин Герман Борисович - руководитель по новым медицинским технологиям компании "Росслин Медикал" (эксклюзивный представитель Deep Breeze); тел: (495) 258-15-09; e-mail: zelenin@rosslynmedical. com

Поступила 24.02.09 (с) Коллектив авторов, 2009 удк 616.24-073 